\title{
The Ontogeny of Hippocampus-Dependent Memories
}

\author{
${ }^{(0}$ Flavio Donato, ${ }^{1}{ }^{\circledR}$ Cristina M. Alberini, ${ }^{2}$ Dima Amso, ${ }^{3}$ George Dragoi, ${ }^{4}$ Alex Dranovsky, ${ }^{5}$ and \\ (1) Nora S. Newcombe ${ }^{6}$ \\ ${ }^{1}$ Biozentrum, University of Basel, 4056, Basel, Switzerland, ${ }^{2}$ Center for Neural Science, New York University, 10003, New York, New York, \\ ${ }^{3}$ Department of Psychology, Columbia University, 10025, New York, New York, ${ }^{4}$ Departments of Psychiatry and Neuroscience, Yale University, \\ 06511, New Haven, Connecticut, ${ }^{5}$ Department of Psychiatry, Columbia University, 10032, New York, New York, and ${ }^{6}$ Temple University, 19122 , \\ Philadelphia, Pennsylvania
}

The formation of memories that contain information about the specific time and place of acquisition, which are commonly referred to as "autobiographical" or "episodic" memories, critically relies on the hippocampus and on a series of interconnected structures located in the medial temporal lobe of the mammalian brain. The observation that adults retain very few of these memories from the first years of their life has fueled a long-standing debate on whether infants can make the types of memories that in adults are processed by the hippocampus-dependent memory system, and whether the hippocampus is involved in learning and memory processes early in life. Recent evidence shows that, even at a time when its circuitry is not yet mature, the infant hippocampus is able to produce long-lasting memories. However, the ability to acquire and store such memories relies on molecular pathways and network-based activity dynamics different from the adult system, which mature with age. The mechanisms underlying the formation of hippocampus-dependent memories during infancy, and the role that experience exerts in promoting the maturation of the hippocampus-dependent memory system, remain to be understood. In this review, we discuss recent advances in our understanding of the ontogeny and the biological correlates of hippocampusdependent memories.

Key words: critical periods; development; early-life stress; hippocampus; learning and memory; place cell sequences

\section{Introduction}

Research on cognitive neuroscience has often considered the infant brain to be an immature version of an adult one, endowed with rudimentary neural networks and limited cognitive abilities. However, although the infant brain is far from mature, it has a remarkable capacity for plasticity and learning and is able to support sophisticated cognitive operations. Babies distinguish words before understanding language (Dehaene-Lambertz et al., 2002), perceive quantity before learning math (Lourenco and Longo, 2010), and perform statistical inference to build expectations about the future (Xu and Garcia, 2008; Teglas et al., 2011). Against this background of cognitive potential, it is arguably surprising that adults retain very few memories of events that happened during the first years of their life, despite the fact that these events shape development. This phenomenon, for which Freud used the expression "infantile amnesia" (Freud, 1914), has sparked a century-long debate on whether infants can make the types of memories that, in adults, are processed by the hippocampus-dependent memory system (i.e., "episodic memories"),

Received June 30, 2020; revised 0ct. 16, 2020; accepted 0ct. 20, 2020.

This work was supported by European Research Council Starter Grant ERC-ST2019 850769 to F.D.; National Institutes of Health F31HD090872 to N.S.N.; National Institute of Neurological Disorders and Stroke R01NS104917 to G.D.; National Institute of Mental Health R01MH121372 to G.D. and MH106809 and MH091844 to A.D.; and DANA foundation MH065635 to C.M.A.

The authors declare no competing financial interests.

Correspondence should be addressed to Flavio Donato at flavio.donato@unibas.ch.

https://doi.org/10.1523/JNEUROSCI.1651-20.2020

Copyright $(2021$ the authors and whether the hippocampus is involved in learning and memory processes early in life.

Psychology-centered views hypothesized that the rapid forgetting of childhood events is caused by the absence of advanced cognitive constructs, such as the sense of self, theory of mind, or language (Perner and Ruffman, 1995). Biological theories, on the other hand, were built on the observation that infantile amnesia also appears in nonhuman species (Campbell and Campbell, 1962) and postulated that amnesia stems from the developing hippocampus' limited ability to process information for encoding, storing, and recalling memories, most likely because of delayed or ongoing maturation of its circuitry (for an extensive review, see Josselyn and Frankland, 2012). In support of the biological hypothesis, Akers et al. (2014) demonstrated that elevated rates of neurogenesis in the postnatal hippocampus contributed to the rapid forgetting of memories formed during infancy, and that reducing neurogenesis after memory acquisition at such age could increase the persistence of these hippocampus-dependent memories. Fundamental questions were raised: when does the hippocampus-dependent memory system acquire the ability to represent experience-dependent information? When does it become competent to perform what kind of learning? And what is the destiny of hippocampus-dependent memories formed during infancy?

While there is reason to believe that complex forms of episodic memory are not formed early during life, there is also very good reason to suspect that some hippocampus-dependent learning and memory mechanisms are indeed available to infants. In rodents, hippocampal neurons are already able to 
tune their activity to experience-dependent variables during infancy (Langston et al., 2010; Wills et al., 2010), and can create long-lasting biological changes supporting synapse maturation where memories of early-life events are encoded (Travaglia et al., 2016; Bessières et al., 2020). In humans, it has been more difficult to assess this issue. Findings, such as the fact that months-old infants show the effects of playing with a specific mobile or seeing a specific face for up to 2 weeks (Rovee-Collier, 1999), stumble on a lack of knowledge as to whether such memories are mediated by the hippocampus. These discoveries have set in motion a paradigm shift in the way we think about the ontogeny of hippocampus-dependent memories: far from being incompetent to learn, the hippocampus already has the ability to support the formation of rich representations and long-lasting memories during infancy, although its ability to learn from experience, its computational algorithms, and its circuitry, mature with age.

In this review, we will present a series of studies whose aim is to gain a mechanistic understanding of the ontogeny of hippocampus-dependent memories in rodents and humans, and to integrate information gathered at multiple levels of investigation, from the cognitive to the molecular. First, we will define behavioral aspects of cognitive development in humans, to highlight the functional properties of memories created during infancy and assess their dependence on the hippocampus. Second, we will look into the structural and functional maturation of the hippocampal network and its neural code in rodents, to identify the computational algorithms by which the infant hippocampus works. Third, we will focus on neurons and their connections to understand how memory processes are executed by the developing rodent hippocampus, how memories of early-life experiences are stored long-term, and how they affect the development of hippocampus-dependent cognitive functions.

\section{Hippocampus-dependent memories during infancy and childhood}

The study of the ontogeny of memory has occurred within the context of theoretical debates about the value of implementing a multiple memory systems framework to infant cognitive processes (Richmond and Nelson, 2007). According to this framework, the brain is endowed with distinct memory systems which differ in their neurobiological substrates and rules of operation (Sherry and Schacter, 1987), with separate systems for the production of explicit (declarative) and implicit (procedural) memories (Squire, 1992). Within the explicit memory system, there is an additional distinction between memories for the static knowledge of semantic facts, and the temporal and spatial particularities of autobiographical experiences (i.e., the "context" of episodic memories). The first would largely depend on cortical structures of the medial temporal lobe, while the second would critically rely on an intact hippocampus (Vargha-Khadem et al., 1997). While there is evidence of both implicit memory and long-term retention of learned contextual information in human infants (Rovee-Collier and Cuevas, 2009; Cuevas and Sheya, 2019), what constitutes an episode that can be consciously recollected in a nonverbal infant is unclear. Some have argued that human infants can form episodic memories by the end of the first postnatal year, while others argue that relational memories built during infancy are not episodic, but semantic in nature, and are likely cortically mediated (Olson and Newcombe, 2014; Gomez and Edgin, 2016).

Some of the initial clues about the ontogeny of hippocampusdependent memories came from research on spatial memory tasks and contextually contingent binding, which demonstrated that place learning (which requires the hippocampus) could be first seen at $\sim 21$ months of age (Newcombe et al., 1998; Balcomb et al., 2011). Newcombe et al. (2014) let children explore two rooms, each of them endowed with the same four containers arranged in different configurations. One container in each room opened to reveal a distinctive toy, and the others were empty. When toddler and preschoolers were prompted to search for a specific toy, Newcombe et al. (2014) found that toddlers performed at chance level until after 20 months, even with a potent retrieval cue and a very short delay period between exploration and retrieval.

More recently, researchers have used nonverbal readouts to investigate the ability to extract contextual knowledge of the world in even younger infants. Specifically, Tummeltshammer and Amso (2018) used eye tracking and contextual cueing tasks to investigate whether infants could acquire and use contextual information to guide attention during visual search. The authors embedded target objects in repeated versus novel spatial scene contexts, and let infants simply scan the screen as they presented interleaved trials. Their results indicated that 6- and 10-monthold infants were better able to detect the target object, measured by a correct eye movement to its location, when the spatial context in which it appeared was repeated than when the context was variable or novel, thereby demonstrating that infants can use contextual information to facilitate orienting in a memoryguided visual task.

The ability to perform nonspatial, hippocampus-dependent tasks develops during the same period. Deferred imitation of action sequences first appears at 6 months, but only in simple forms involving short sequences, brief delays, or repeated retrievals (Barr et al., 1996, 2005; Adlam et al., 2005). Over the first 2 years of life, children gain the ability to encode longer sequences, show longer retention, and require less specific cues for retrieval (Bauer et al., 1994; Hayne et al., 2000). Crucially, it is only in the second year of life that children begin to exhibit the ability to encode and retain an arbitrary temporal sequence of actions, at 22 months on an immediate test, and at 28 months after a 2 week delay (Bauer et al., 1998).

Studying the neural correlates of learning and memory in human infants is plagued by methodological limitations that have hindered our understanding of whether the robust learning and memory skills exhibited by infants are hippocampally mediated. The primary methodological limitation is in the imaging technology available for testing awake and behaving infants. Functional imaging tools regularly used in infancy, including functional near infrared spectroscopy, are only valuable for recording cortical activity. fMRI has been used extensively in resting state connectivity studies in sleeping infants (e.g., Power et al., 2011). However, robust signals in response to learning and memory tasks in awake human infants in the scanner remain difficult.

The earliest evidence of hippocampal activation was recorded in children that were 25 months or older (Prabhakar et al., 2018). In this study, Prabhakar et al. (2018) studied hippocampal activation during natural sleep in toddlers who had previously heard a novel song in a distinctive context and with a distinctive toy. The results indicated that toddlers showed greater hippocampal activation during a sleep session when the previously heard song was played, than during a control song never heard before. This activation effect was especially marked for toddlers who were able to indicate the correct room and toy character associated with the song. Thus, it seems likely that the observed hippocampal activity involved reactivation of an episodic memory. Of 
a

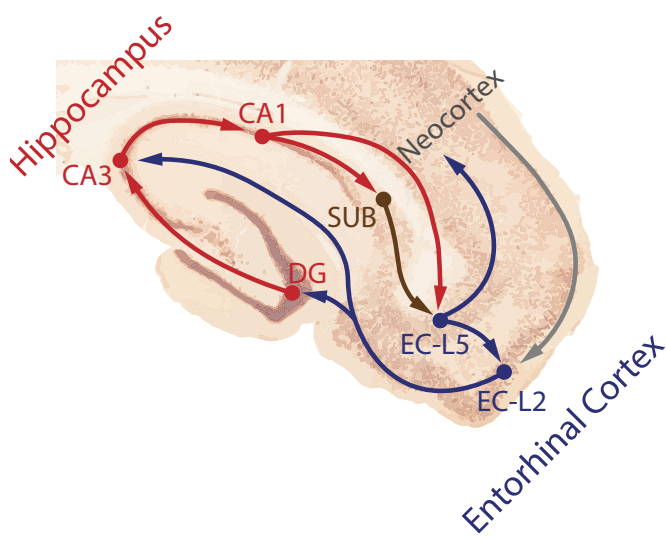

b

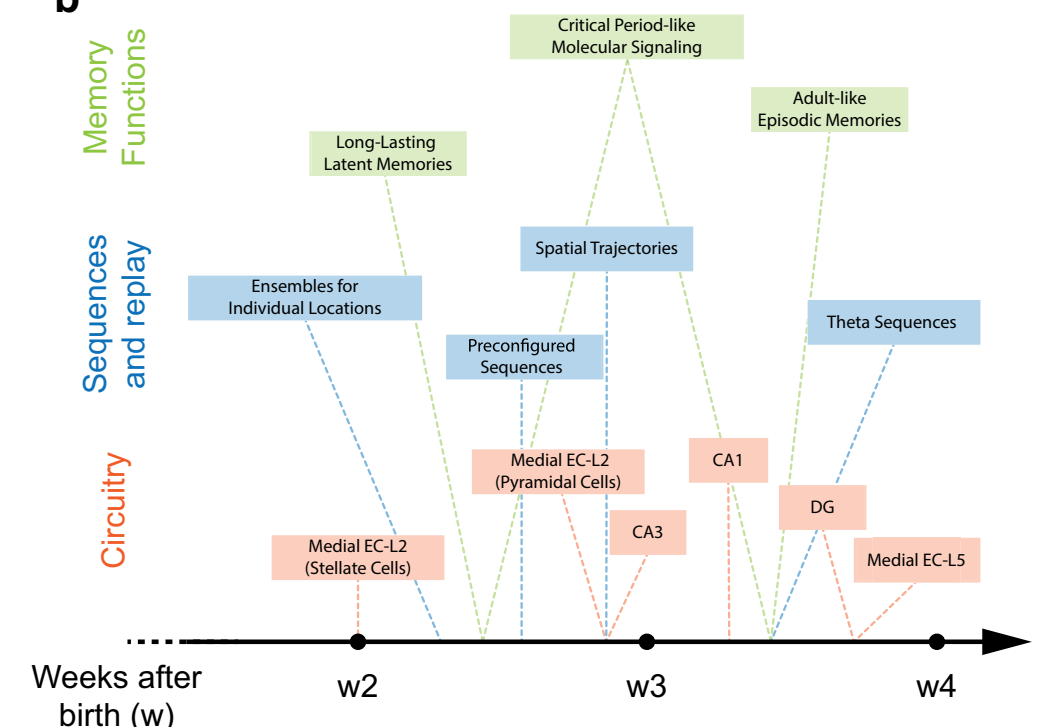

Figure 1. $\quad \boldsymbol{a}$, Schematic representation of the entorhinal-hippocampal circuit in the rat. Sensory information reaches the three regions of the hippocampus (dentate gyrus [DG], CA3, and CA1) via the superficial layers of the entorhinal cortex (EC-L2). In turn, the output station CA1 and subiculum (SUB) project back to the deep layers of the entorhinal cortex (EC-L5), which broadcasts the hippocampal output to multiple cortical areas. $\boldsymbol{b}$, A comparative timeline delineating relevant milestones in the structural and functional maturation of the hippocampus-dependent memory system of rodents.

course, these children were already 2 years or older, and hence beyond the boundary of infantile amnesia and at the start of the period during which it is believed that hippocampus-dependent episodic memory formation is possible. It would be fascinating to expand the application of this procedure to infants, but this will require the development of new techniques for measuring subcortical activity at these ages. Animal models, on the contrary, provide the opportunity to overcome these limitations and record the activity of hippocampal neurons at single-cell resolution during development. We will discuss such studies in the next section.

\section{A protracted developmental trajectory for the hippocampal network and the emergence of its neural code}

The hippocampal network comprises several interconnected areas located within the hippocampus proper (i.e., dentate gyrus, the CA fields, and the subiculum) and the parahippocampal complex (i.e., medial and lateral entorhinal cortex). Although these areas are directly and indirectly connected through multiple routes, it is generally assumed that, in the adult network, information from sensory and association cortices reaches the hippocampus through the superficial layers of the entorhinal cortex, before being routed toward the deep layers of the parahippocampal complex and broadcasted to the rest of the brain (van Strien et al., 2009) (Fig. 1a). The activity of neurons located in these areas is modulated by an animal's position (as in the case of place and grid cells), orientation in space (as in the case of head-direction cells), or elapsed time (time cells), and supports the creation of a cognitive map of experience for the formation of episodic and semantic memories (Moser et al., 2015). Through the years, multiple theories have been proposed to explain the mechanistic contribution of the hippocampus to episodic memory processes. One theory postulated that, by representing information about space and time, the hippocampal network provides a spatiotemporal context to episodic events (O'Keefe and Nadel, 1978). Alternatively, the hippocampal code might work as an index that points to experience-related information stored in the neocortex (Teyler and Discenna, 1986). Either way, the hippocampus is essential for the relational binding of spatial locations and events into spatial and mental trajectories and memory episodes (Eichenbaum and Cohen, 2014). The rapid encoding and consolidation of sequential experiences into memory episodes are believed to be achieved by the representation of such trajectories in the sequential firing of neurons, which appear to encode specific locations in space, the "place cells," and the activation of such place cells within timecompressed sequences during navigation (Dragoi and Buzsáki, 2006), and during the sleep/rest periods preceding (i.e., preplay, supporting rapid encoding) (Dragoi and Tonegawa, 2011) and following (i.e., replay, supporting consolidation) (Lee and Wilson, 2002) a novel experience. Importantly, the hippocampus is able to handle incoming sensory information in an associative fashion to avoid interference between memories. Distinct ensembles and sequences of place cells are recruited on distinct contexts (pattern separation), whereas similar ensembles and sequences are recruited on repeated exposures to the same context, even when only incomplete sensory information about that context is available (pattern completion) (Guzowski et al., 1999; Leutgeb et al., 2007).

Hippocampal circuits mature late during brain development compared with other cortical areas, with distinct regions and subfields maturing at different rates (Sowell et al., 2003; Deguchi et al., 2011; Gomez and Edgin, 2016). While this late developmental trajectory is observed in both humans and rodents, a notable difference between the two species is that the first 2 weeks of postnatal development in rodents correspond to the latter part of pregnancy in human brain development. Moreover, neurodevelopmental milestones associated with the end of infancy in humans (2- to 3-year-olds) are reached by the end of the third postnatal week in rodents, whereas those demarking the end of childhood in humans (9- to 11-year-olds) roughly correspond to the end of the first month of life of a rat or a mouse (Semple et al., 2013; Workman et al., 2013).

In humans, the volume of the hippocampal formation increases sharply until the age of 2 and continues to increase slowly thereafter 
up to the age of 12 (Utsunomiya et al., 1999). This increase is mostly attributable to the addition of newly born neurons in the dentate gyrus, synaptogenesis, and myelination of axonal fibers. A similarly protracted course of development has been observed in rodents, where Donato et al. (2017) used the expression of histologic markers associated with neurogenesis and synaptogenesis to create a spatiotemporal map describing the maturation of the entorhinal-hippocampal network at single-cell resolution during the first month of life of a mouse (Fig. 1b). In that study, the authors revealed a stepwise progression of maturation that followed the same sequence through which information propagates in the adult rodent circuit and was mechanistically driven by an excitatory activity-associated signal propagating synaptically across the network. The propagation of the maturationdriving signal defined a developmental hierarchy that saw the superficial layers of the medial entorhinal cortex at the top, the hippocampus in the middle, and the deep layers of the entorhinal cortex at the bottom. Silencing excitatory activity at any stage of the network impaired maturation in the areas downstream in the hierarchy, but not in those upstream, where maturation progressed to adult-like levels. Surprisingly, the maturation of stellate cells in the superficial medial entorhinal cortex was not affected by silencing and proceeded with a time course that correlated with neurogenesis. Because the activation of these neurons was necessary for the maturation of other neuronal populations within the entorhinal-hippocampal circuit, the authors proposed that stellate cells could function as a cellautonomous "driver" for the development of the hippocampal network (Donato, 2017).

The emergence of spatial tuning in the activity of hippocampal and entorhinal neurons does not follow the same sequence as the network"s structural maturation, despite largely happening during the same postnatal time window. In a series of landmark studies conducted in rodents, it was shown that head-direction cells in the parahippocampal cortex emerge first, followed by place cells in the hippocampus, with grid cells in the entorhinal cortex emerging last (Langston et al., 2010; Wills et al., 2010). One important point to be made though is that individual cell's tuning to space did not exhibit the same degree of specificity, stability, or information content expressed in the adult at the onset of spatial exploration, but underwent progressive maturation and refinement. Importantly, hippocampal place cells exhibited associative-memory dynamics (i.e., were able to perform pattern completion and separation) already in juvenile rats (Muessig et al., 2016).

Memories are thought to be formed in several stages (Buzsáki, 1989). In adult rodents, encoding of spatial representations is associated with binding of adjacent past-current-future locations by temporally compressed sequences of place cells (i.e., theta sequences) nested within theta oscillation. During the consolidation of spatial representations, these experience-related compressed sequences are replayed during postexperience sleep primarily in association with ripple oscillations, and at higher incidence and precision compared with their preplay during preexperience sleep (the higher incidence of replay vs preplay is known as "plasticity in replay") (Farooq et al., 2019). Several studies have shown that the main network oscillatory patterns (theta and ripples) are present at the beginning of the third postnatal week in the rat hippocampus (Buhl and Buzsáki, 2005; Farooq and Dragoi, 2019; Muessig et al., 2019). It was, however, still not known when and how, during postnatal brain maturation, the ensemble sequential firing patterns emerged, whether the spatial tuning and theta sequences emerged in coordination, and whether any form of prior spatial experience was necessary and sufficient to induce the striking repertoire of compressed temporal sequence motifs observed in adulthood. Recently, Farooq and Dragoi (2019) described three distinct stages in the postnatal development of time-compressed neuronal sequences of firing in the rat CA1 after eye opening (Fig. $1 b$ ). Stage 1 ended around postnatal day 16 (P16) and was characterized by the presence of neuronal ensembles whose activity depicted individual locations in the environment, but not sequential trajectories before, during, or after the navigational experience. Spontaneous preconfigured sequences (sleep preplay) and rapid encoding of novel navigational trajectories at clock-timescale during navigation, but no detectable theta sequences and plasticity in replay, emerged gradually around P17-P18 and appeared to support encoding of full trajectories only at P19-P20, during developmental Stage 2. During Stage 3, starting around P23-P24, sequentially experienced locations in the environment were uniquely bound into larger trajectories within hippocampal theta sequences during navigation, and were subsequently replayed during sleep at higher incidence and precision compared with their preplay. Both theta sequence compression and navigation-related plasticity in replay emerged in coordination from spontaneous preconfigured sequences over the course of the fourth postnatal week.

These studies revealed a protracted developmental time course for the construction of the hippocampal circuitry, and a progressive refinement of the rodent hippocampal neural code that culminated with the emergence of theta sequences and the precise representation of past, present, and future locations into a single spatial trajectory (Fig. $1 b$ ). It is tempting to speculate that similar phenomena might support the development of a child's ability to bind past, current, and future events into a single mental trajectory, which might support the developing competence in imitating action sequences. Fascinatingly, both structural and functional data converged on the hypothesis that the assembly of the hippocampal network, and the emergence of ensembles' sequential firing, might be initiated by age-dependent internal developmental programs. However, the mechanisms and factors implicated in the transition of neuronal ensemble patterns between the three developmental stages are still unknown, and the role that experience plays in the structural and functional maturation of the hippocampus-dependent memory system remains to be determined.

\section{Experience-dependent maturation of hippocampus- dependent memory functions}

Although early-life experiences appear to be rapidly forgotten, they are critically important for the development of personality and cognitive abilities, and they can affect brain functions throughout life. Recently, thanks to the development of novel molecular methodologies by which we can study populations of active neurons and manipulate their activity in laboratory animals, a series of studies has been able to shed light on the mechanisms and circuitry by which early life experiences influence the physiological development of hippocampus-dependent memory functions in rodents.

More specifically, Travaglia et al. (2016) were able to show that an episodic aversive memory formed in infancy, despite not being expressed at the behavioral level when the animal was reexposed to the conditioned stimulus, was not lost, but it was stored as a long-term representation in a latent modality, and could be reinstated by an appropriate behavioral reminder presented up to 4 weeks after the memory appeared to be forgotten 
(Fig. 1b). Subsequent experiments in both aversive and nonaversive episodic learning paradigms showed that the formation and storage of infant memories in a latent form required the hippocampus (Travaglia et al., 2018). Molecular characterization of hippocampal changes evoked by infantile learning revealed that learning recruited unique mechanisms and molecular pathways in the hippocampus of infant rats and mice compared with juveniles and adults. These mechanisms overlapped with those that had previously been discovered to be engaged during sensory critical periods, and they included a BDNF- and metabotropic glutamate receptor-5-dependent switch in the expression of the NMDAR subunits, from 2B to 2A (Travaglia et al., 2016). Learning-induced changes also included persistent neuronal activation measured by induction of immediate early genes, BDNF-dependent increases in the excitatory synapse markers synaptophysin and PSD-95, and significant maturation of AMPAR synaptic responses (Bessières et al., 2020). These data led to the conclusions that (1) the hippocampus, like sensory systems, undergoes a developmental critical period to become functionally competent; and (2) on learning a specific stimulus/context association, the infant hippocampus has heightened neuronal activation that follows a distinct kinetic from the adult hippocampus, and can last for up to $48 \mathrm{~h}$ after learning.

Along the same lines, Guskjolen et al. (2018) used the promoter for the immediate early gene Arc to drive the brainwide expression of Channelrohodpsin2 in ensembles of neurons that were activated during infant memory acquisition, and showed that "forgotten" infant memories could be rescued in adult animals by the direct optogenetic reactivation of Channelrohodpsin2-labeled ensembles located in the hippocampus. Furthermore, Bessières et al. (2020) found that presenting a second, distinct learning experience (i.e., the association of a novel stimulus/context association) during the period of learninginduced heightened activation of the hippocampus led to the maturation of memory functional competence (i.e., the ability to express memories long-term) (Bessières et al., 2020). This process was selective for the type of experience the animal had, as the experience-dependent maturation transferred only to similar hippocampus-dependent learning domains (e.g., within contextual aversive similar types of learning), but not to other types of hippocampus-dependent learning (e.g., from contextual aversive learning to spatial nonaversive learning).

Together, these studies strongly supported the conclusion that, in infancy, episodic learning activates and functionally engages the hippocampus-dependent memory system to form and store memories that persist in a latent form long-term and through adulthood. Moreover, they revealed that infantile learning biologically matures the hippocampus, and it does so in an experience-specific manner, thereby producing selective maturation of memory abilities.

Not all experiences have the same effect on the maturation of hippocampus-dependent cognitive functions. For example, stressful experiences have lasting effects on the hippocampus; and when they occur during early life, their consequences often persist in shaping behavior for years to come. The mechanism by which early life adversity causes more persisting hippocampal changes than its later counterpart has been studied on multiple levels. Adult animals exposed to experimental models of early life stress exhibit, among other things, changes in hippocampusdependent behaviors, decreased hippocampal volume, dysregulation of the hypothalamic-pituitary-adrenal axis, and changes in gene regulation that might be produced by an altered pattern of genome methylation and acetylation (McEwen, 2020). While the link between early adversity and molecular changes is compelling, a strong causal link between such changes and adult behavior has yet to be established. Hence, the mechanisms by which early life stress impacts circuits governing adult behavior remain elusive.

In addition to causing morphologic changes, chronic stress strongly affects the adult dentate gyrus stem-cell system. Stress exposure directs neural stem cells toward self-renewal (Bonaguidi et al., 2011; Dranovsky et al., 2011) and is a potent suppressor of neurogenesis (Snyder and Drew, 2020). Moreover, ongoing neurogenesis appears to play a direct role in adaptation to stress (Snyder et al., 2011; Dranovsky and Leonardo, 2012). The possibility that sustained effects of early life stress can be encoded by the stem-cell system is especially intriguing because most of the adult dentate gyrus is formed during the first several weeks of life in a rodent (Bayer and Altman, 1974). Thus, any adverse experiences during this sensitive period can alter the developmental trajectory of the dentate gyrus. Remarkably, some reports have suggested that early life stress exposure is associated with a paradoxical increase in neurogenesis at the end of the exposure (Naninck et al., 2015). A recent study examining the effects of P3-P10 stress over time revealed that what appears as neurogenic effects of early life stress actually reflects a delay in the maturation of the dentate gyrus, thus providing a potential explanation for the apparent paradox (Youssef et al., 2019).

Given the stress sensitivity of dentate gyrus development and its largely postnatal course, it is intriguing to speculate that the precise timing of stress exposure can lead to diverse hippocampal phenotypes. Indeed, briefly suppressing neurogenesis during early adolescence, but not in adulthood, results in stress resilience (Kirshenbaum et al., 2014). Earlier periods of neurogenesis suppression revealed separate sensitive periods for establishing the adult stem cell pool and homeostatic set points for adult neurogenesis (Youssef et al., 2018). Together, the results suggest that the developing dentate gyrus is a "moving target" of sensitivity to insults that can result in diverse and persisting consequences for adult hippocampal function. Both the molecular and circuit mechanisms that determine how developmental sensitivity translates to sustained hippocampal changes remain to be determined.

\section{Looking ahead}

The past decade has been tremendously exciting for researchers interested in understanding the ontogeny of hippocampus-dependent memories. We have been able to record functional signal of hippocampal engagement during memory tasks in human toddlers. We have observed the emergence of the cognitive map in the developing rodent medial temporal lobe. We have dissected the processes that lead to the assembly of the extended hippocampal-entorhinal circuit in mice. We have been able to study the molecular and cellular substrates of infant memories in the developing rodent brain. The series of landmark discoveries highlighted in this review have provided strong evidence suggesting that (1) the developing hippocampus is engaged in learning and memory processes already during infancy; (2) memories created by the infant hippocampus rely on molecular pathways and network-based activity dynamics that are distinct from those operating during adulthood; and (3) the hippocampus undergoes a developmental critical period for learning to learn. However, we still fall short of understanding the network mechanisms underlying the formation of hippocampus-dependent memories during early development, the impact that infant memories exert on cognitive function later in life, and the extent to which the 
same biological underpinnings support the ontogeny of hippocampus-dependent memories across species. Looking ahead, it is time to focus our efforts on providing a comprehensive framework that allows us to link the molecular responses induced by learning to the emergence of coordinated firing patterns in the developing rodent hippocampus, and to compare the developmental trajectory of cognitive functions between rodents and humans. To tackle these questions, we advocate for a multidisciplinary approach for the creation of a long-needed bridge between developmental systems and cognitive neuroscience, and for the application of this approach across the evolutionary spectrum. The success of such operation will depend on establishing paradigms that are useful across ages and species, evolving new techniques for studying neural activity in young humans, and tackling issues with behavioral work with the fast-developing rodent, perhaps through controlled rearing experiments. Our hope is that dissecting the ontogeny of hippocampus-dependent memories might open new ways of thinking about how the brain is built and functions, and might change our approach to children's education and the understanding and treatment of neurodevelopmental disorders.

\section{References}

Adlam AL, Vargha-Khadem F, Mishkin M, de Haan M (2005) Deferred imitation of action sequences in developmental amnesia. J Cogn Neurosci 17:240-248.

Akers KG, Martinez-Canabal A, Restivo L, Yiu AP, De Cristofaro A, Hsiang HL, Wheeler AL, Guskjolen A, Niibori Y, Shoji H, Ohira K, Richards BA, Miyakawa T, Josselyn SA, Frankland PW (2014) Hippocampal neurogenesis regulates forgetting during adulthood and infancy. Science 344:598602.

Balcomb F, Newcombe NS, Ferrara K (2011) Finding where and saying where: developmental relationships between place learning and language in the first year. J Cogn Dev 12:315-331.

Barr R, Dowden A, Hayne H (1996) Developmental changes in deferred imitation by 6- to 24-month-old infants. Infant Behav Dev 19:159-170.

Barr R, Rovee-Collier C, Campanella J (2005) Retrieval protracts deferred imitation by 6-month-olds. Infancy 7:263-283.

Bauer PJ, Hertsgaard LA, Dow GA (1994) After 8 months have passed: longterm recall of events by 1 -year-old to 2 -year-old children. Memory 2:353-382.

Bauer PJ, Hertsgaard LA, Dropik P, Daly BP (1998) When even arbitrary order becomes important: developments in reliable temporal sequencing of arbitrarily ordered events. Memory 6:165-198.

Bayer SA, Altman J (1974) Hippocampal development in the rat: cytogenesis and morphogenesis examined with autoradiography and low-level Xirradiation. J Comp Neurol 158:55-79.

Bessières B, Travaglia A, Mowery TM, Zhang X, Alberini CM (2020) Early life experiences selectively mature learning and memory abilities. Nat Commun 11:628.

Bonaguidi MA, Wheeler MA, Shapiro JS, Stadel RP, Sun GJ, Ming GL, Song $\mathrm{H}$ (2011) In vivo clonal analysis reveals self-renewing and multipotent adult neural stem cell characteristics. Cell 145:1142-1155.

Buhl DL, Buzsáki G (2005) Developmental emergence of hippocampal fastfield "ripple" oscillations in the behaving rat pups. Neuroscience 134: $1423-1430$.

Buzsáki G (1989) Two-stage model of memory trace formation: a role for "noisy" brain states. Neuroscience 31:551-570.

Campbell BA, Campbell EH (1962) Retention and extinction of learned fear in infant and adult rats. J Comp Physiol Psychol 55:1-8.

Cuevas K, Sheya A (2019) Ontogenesis of learning and memory: biopsychosocial and dynamical systems perspectives. Dev Psychobiol 61:402-415.

Deguchi Y, Donato F, Galimberti I, Cabuy E, Caroni P (2011) Temporally matched subpopulations of selectively interconnected principal neurons in the hippocampus. Nat Neurosci 14:495-504.

Dehaene-Lambertz G, Dehaene S, Hertz-Pannier L (2002) Functional neuroimaging of speech perception in infants. Science 298:2013-2015.
Donato F (2017) Assembling the brain from deep within stellate cells appears to drive the maturation of the entorhinal-hippocampal network. Science 358:456-457.

Donato F, Jacobsen RI, Moser MB, Moser EI (2017) Stellate cells drive maturation of the entorhinal-hippocampal circuit. Science 355:eaai8178.

Dragoi G, Buzsáki G (2006) Temporal encoding of place sequences by hippocampal cell assemblies. Neuron 50:145-157.

Dragoi G, Tonegawa S (2011) Preplay of future place cell sequences by hippocampal cellular assemblies. Nature 469:397-401.

Dranovsky A, Leonardo ED (2012) Is there a role for young hippocampal neurons in adaptation to stress? Behav Brain Res 227:371-375.

Dranovsky A, Picchini AM, Moadel T, Sisti AC, Yamada A, Kimura S, Leonardo ED, Hen R (2011) Experience dictates stem cell fate in the adult hippocampus. Neuron 70:908-923.

Eichenbaum H, Cohen NJ (2014) Can we reconcile the declarative memory and spatial navigation views on hippocampal function? Neuron 83:764770 .

Farooq U, Dragoi G (2019) Emergence of preconfigured and plastic timecompressed sequences in early postnatal development. Science 363:168173.

Farooq U, Sibille J, Liu K, Dragoi G (2019) Strengthened temporal coordination within pre-existing sequential cell assemblies supports trajectory replay. Neuron 103:719-733.e717.

Freud S (1914) Psychopathology of everyday life. New York: The McMillian Company.

Gomez RL, Edgin JO (2016) The extended trajectory of hippocampal development: implications for early memory development and disorder. Dev Cogn Neurosci 18:57-69.

Guskjolen A, Kenney JW, de la Parra J, Yeung BR, Josselyn SA, Frankland PW (2018) Recovery of "lost" infant memories in mice. Curr Biol 28:2283-2290.e3.

Guzowski JF, McNaughton BL, Barnes CA, Worley PF (1999) Environmentspecific expression of the immediate-early gene Arc in hippocampal neuronal ensembles. Nat Neurosci 2:1120-1124.

Hayne H, Boniface J, Barr R (2000) The development of declarative memory in human infants: age-related changes in deferred imitation. Behav Neurosci 114:77-83.

Josselyn SA, Frankland PW (2012) Infantile amnesia: a neurogenic hypothesis. Learn Mem 19:423-433.

Kirshenbaum GS, Lieberman SR, Briner TJ, Leonardo ED, Dranovsky A (2014) Adolescent but not adult-born neurons are critical for susceptibility to chronic social defeat. Front Behav Neurosci 8:289.

Langston RF, Ainge JA, Couey JJ, Canto CB, Bjerknes TL, Witter MP, Moser EI, Moser MB (2010) Development of the spatial representation system in the rat. Science 328:1576-1580.

Lee AK, Wilson MA (2002) Memory of sequential experience in the hippocampus during slow wave sleep. Neuron 36:1183-1194.

Leutgeb JK, Leutgeb S, Moser MB, Moser EI (2007) Pattern separation in the dentate gyrus and CA3 of the hippocampus. Science 315:961-966.

Lourenco SF, Longo MR (2010) General magnitude representation in human infants. Psychol Sci 21:873-881.

McEwen BS (2020) Hormones and behavior and the integration of brainbody science. Horm Behav 119:104619.

Moser MB, Rowland DC, Moser EI (2015) Place cells, grid cells, and memory. Cold Spring Harb Perspect Biol 7:a021808.

Muessig L, Hauser J, Wills TJ, Cacucci F (2016) Place cell networks in preweanling rats show associative memory properties from the onset of exploratory behavior. Cereb Cortex 26:3627-3636.

Muessig L, Lasek M, Varsavsky I, Cacucci F, Wills TJ (2019) Coordinated emergence of hippocampal replay and theta sequences during post-natal development. Curr Biol 29:834-840.e4.

Naninck EF, Hoeijmakers L, Kakava-Georgiadou N, Meesters A, Lazic SE, Lucassen PJ, Korosi A (2015) Chronic early life stress alters developmental and adult neurogenesis and impairs cognitive function in mice. Hippocampus 25:309-328.

Newcombe N, Huttenlocher J, Drummey AB, Wiley JG (1998) The development of spatial location coding: place learning and dead reckoning in the second and third years. Cogn Dev 13:185-200.

Newcombe NS, Balcomb F, Ferrara K, Hansen M, Koski J (2014) Two rooms, two representations? Episodic-like memory in toddlers and preschoolers. Dev Sci 17:743-756. 
O'Keefe J, Nadel L (1978) The hippocampus as a cognitive map. New York: Oxford UP.

Olson RI, Newcombe S (2014) Relational memory and the developmental trajectory of the hippocampus. In: The Wiley handbook on the development of children's memory (Bauer PJ, ed). New York: Wiley.

Perner J, Ruffman T (1995) Episodic memory and autonoetic consciousness: developmental evidence and a theory of childhood amnesia. J Exp Child Psychol 59:516-548.

Power JD, Cohen AL, Nelson SM, Wig GS, Barnes KA, Church JA, Vogel AC, Laumann TO, Miezin FM, Schlaggar BL, Petersen SE (2011) Functional network organization of the human brain. Neuron 72:665678.

Prabhakar J, Johnson EG, Nordahl CW, Ghetti S (2018) Memory-related hippocampal activation in the sleeping toddler. Proc Natl Acad Sci USA 115:6500-6505.

Richmond J, Nelson CA (2007) Accounting for change in declarative memory: a cognitive neuroscience perspective. Dev Rev 27:349-373.

Rovee-Collier C (1999) The development of infant memory. Curr Dir Psychol Sci 8:80-85.

Rovee-Collier C, Cuevas K (2009) Multiple memory systems are unnecessary to account for infant memory development: an ecological model. Dev Psychol 45:160-174.

Semple BD, Blomgren K, Gimlin K, Ferriero DM, Noble-Haeusslein LJ (2013) Brain development in rodents and humans: identifying benchmarks of maturation and vulnerability to injury across species. Prog Neurobiol 106:1-16

Sherry DF, Schacter DL (1987) The evolution of multiple memory-systems. Psychol Rev 94:439-454.

Snyder JS, Drew MR (2020) Functional neurogenesis over the years. Behav Brain Res 382:112470.

Snyder JS, Soumier A, Brewer M, Pickel J, Cameron HA (2011) Adult hippocampal neurogenesis buffers stress responses and depressive behaviour. Nature 476:458-461.

Sowell ER, Peterson BS, Thompson PM, Welcome SE, Henkenius AL, Toga AW (2003) Mapping cortical change across the human life span. Nat Neurosci 6:309-315.

Squire LR (1992) Declarative and nondeclarative memory: multiple brain systems supporting learning and memory. J Cogn Neurosci 4:232-243.
Teglas E, Vul E, Girotto V, Gonzalez M, Tenenbaum JB, Bonatti LL (2011) Pure reasoning in 12-month-old infants as probabilistic inference. Science 332:1054-1059.

Teyler TJ, Discenna P (1986) The hippocampal memory indexing theory. Behav Neurosci 100:147-154.

Travaglia A, Bisaz R, Sweet ES, Blitzer RD, Alberini CM (2016) Infantile amnesia reflects a developmental critical period for hippocampal learning. Nat Neurosci 19:1225-1233.

Travaglia A, Steinmetz AB, Miranda JM, Alberini CM (2018) Mechanisms of critical period in the hippocampus underlie object location learning and memory in infant rats. Learn Mem 25:176-182.

Tummeltshammer K, Amso D (2018) Top-down contextual knowledge guides visual attention in infancy. Developmental Sci 21:e12599.

Utsunomiya H, Takano K, Okazaki M, Mitsudome A (1999) Development of the temporal lobe in infants and children: analysis by MR-based volumetry. AJNR Am J Neuroradiol 20:717-723.

van Strien NM, Cappaert NL, Witter MP (2009) The anatomy of memory: an interactive overview of the parahippocampal-hippocampal network. Nat Rev Neurosci 10:272-282.

Vargha-Khadem F, Gadian DG, Watkins KE, Connelly A, Van Paesschen W, Mishkin M (1997) Differential effects of early hippocampal pathology on episodic and semantic memory. Science 277:376-380.

Wills TJ, Cacucci F, Burgess N, O’Keefe J (2010) Development of the hippocampal cognitive map in preweanling rats. Science 328:1573-1576.

Workman AD, Charvet CJ, Clancy B, Darlington RB, Finlay BL (2013) Modeling transformations of neurodevelopmental sequences across mammalian species. J Neurosci 33:7368-7383.

Xu F, Garcia V (2008) Intuitive statistics by 8-month-old infants. Proc Natl Acad Sci U S A 105:5012-5015.

Youssef M, Atsak P, Cardenas J, Kosmidis S, Leonardo ED, Dranovsky A (2019) Early life stress delays hippocampal development and diminishes the adult stem cell pool in mice. Sci Rep 9:4120.

Youssef M, Krish VS, Kirshenbaum GS, Atsak P, Lass TJ, Lieberman SR, Leonardo ED, Dranovsky A (2018) Ablation of proliferating neural stem cells during early life is sufficient to reduce adult hippocampal neurogenesis. Hippocampus 28:586-601. 